\title{
FIXED POINT THEOREMS IN REFLEXIVE BANACH SPACES
}

\author{
R. KANNAN
}

ABstract. In this paper fixed point theorems are established first for mappings $T$, mapping a closed bounded convex subset $K$ of a reflexive Banach space into itself and satisfying

$$
\|T x-T y\| \leqq \frac{1}{2}\{\|x-T x\|+\|y-T y\|\}, \quad x, y \in K,
$$

and then an analogous result is obtained for nonexpansive mappings giving rise to a question regarding the unification of these theorems.

Let $X$ be a reflexive Banach space and let $K$ be a nonempty bounded closed and convex subset of $X$. In [10] Kirk proved the following theorem: If $T$ be a nonexpansive mapping of $K$ into itself i.e., $\|T x-T y\| \leqq\|x-y\|$, $x, y \in K$, and if $K$ has normal structure, then $T$ has a fixed point in $K$. This result was also proved in a uniformly convex space $X$ by Browder [2], Göhde [4] and Goebel [5], the reflexivity of the space and the normal structure of $K$ being consequences of the uniform convexity of $X$.

In this paper first we establish some fixed point theorems for mappings $T$ of $K$ into itself which satisfy

$$
\|T x-T y\| \leqq \frac{1}{2}\{\|x-T x\|+\|y-T y\|\}, \quad x, y \in K .
$$

Mappings $T$ of this type will be referred to as having property A over $K$. Such mappings have been used to study fixed point and other allied problems in [6], [7], [8], [9]. Then we obtain a theorem, analogous to the one proved for a mapping $T$ having property $A$ over $K$, for nonexpansive mappings. We conclude the paper with some observations on this last theorem.

Before going to the theorems, we first recollect the following definitions.

Definition 1 (Normal Structure [10]). A bounded convex set $K$ in a Banach space $X$ is said to have normal structure if for each convex subset $S$ of $K$ which contains more than one point, there exists $x \in S$ such that $\sup _{y \in S}\|x-y\|<\delta(S), \delta(S)$ being the diameter of $S$.

DEFINITION 2 [9]. A mapping $T$ of a bounded subset $K$ of a Banach space $X$ into itself is said to have property B on $K$ [9] if for every closed

Received by the editors August 27, 1971 and, in revised form, June 30, 1972.

AMS (MOS) subject classifications (1970). Primary 47H10; Secondary 54H25.

(c) American Mathematical Society 1973 
convex subset $F$ of $K$, mapped into itself by $T$ and containing more than one element, there exists $x \in F$ such that $\|x-T x\|<\sup _{y \in F}\|y-T y\|$.

It has been shown in [9] that if $K$ has normal structure then a mapping $T$, having property $A$ on $K$, of $K$ into itself must have property B on $K$ but not conversely.

Definition 3. If $T$ is a mapping of $K$ into itself such that for each $x \in K$, $\lim _{n} \delta\left[O\left(T^{n} x\right)\right]<\delta[O(x)]$ when $\delta[O(x)]>0$, where $O\left(T^{r} x\right)=\left\{T^{r} x, T^{r+1} x\right.$, $\cdots\}, r \geqq 0, T^{0} x=x$, then $T$ is said to have diminishing orbital diameters over $K[\mathbf{1}]$.

Throughout this paper, unless otherwise mentioned, $X$ is a reflexive Banach space and $K$ a nonempty bounded closed convex subset of $X$.

We now prove our theorems.

In [8] the following theorem was proved.

THEOREM. Let $T$ be a mapping of a nonempty bounded closed and convex set $K$ of a reflexive Banach space $X$ into itself and let $T$ have property $\mathrm{A}$ over $K$. Then if $\sup _{y \in F}\|y-T y\| \leqq \delta(F) / 2$ for every closed convex bounded subset $F$ of $K$ mapped into itself by $T, T$ must have a unique fixed point in $K$.

We now prove the following theorem which is obviously an improvement on the preceding result.

THEOREM 1. Let $T$ be a mapping of a nonempty bounded closed and convex subset $K$ of a reflexive Banach space $X$ into itself and let $T$ have property A over $K$. Then if $\sup _{y \in F}\|y-T y\|<\delta(F)$ for every nonempty bounded closed convex subset $F$ of $K$, containing more than one element and mapped into itself by $T, T$ has a unique fixed point in $K$.

Proof. Smulian [11] has characterized a reflexive Banach space as follows: $X$ is reflexive if and only if every decreasing sequence of nonempty bounded closed convex subsets of $X$ has a nonempty intersection.

Let $\Gamma$ be the family of all closed convex bounded subsets of $K$, mapped into itself by $T$. Obviously $\Gamma$ is nonempty. Applying Zorn's lemma, we get a minimal element $S$ in $\Gamma, S$ being minimal with respect to being nonempty, bounded closed and convex and invariant under $T$. If $S$ contains only one element, then that element is a fixed point of $T$. If not, let $S$ contain more than one element.

Now for $x, y \in S$,

$$
\|T x-T y\| \leqq \frac{\|x-T x\|}{2}+\frac{\|y-T y\|}{2} \leqq \sup _{y \in S}\|y-T y\| .
$$

Hence, $T(S)$ is contained in the closed sphere $C$ with $T x$ as centre and $\sup _{y \in S}\|y-T y\|$ as radius. Also $S \cap C$ is invariant under $T$. Therefore by the minimality of $S$ it follows that $S \subset C$ i.e., $\|T x-y\| \leqq \sup _{y \in S}\|y-T y\|$, for 
every $y \in S$. Hence, for any arbitrary but fixed $x \in S$, we have

$$
\sup _{y \in S}\|T x-y\| \leqq \sup _{y \in S}\|y-T y\| \text {. }
$$

Let $S^{\prime}=\left\{z \in S: \sup _{y \in S}\|z-y\| \leqq \sup _{y \in S}\|y-T y\|\right\}$. Obviously $S^{\prime}$ is closed, convex and nonempty $\left(T x \in S^{\prime}\right)$. Again if $z \in S^{\prime}$, then $z \in S$ and hence $T z \in S^{\prime}$ by (1). Hence $S^{\prime}$ is invariant under $T$. Also

$$
\delta\left(S^{\prime}\right) \leqq \sup _{y \in S}\|y-T y\|<\delta(S) \text { by hypothesis. }
$$

Hence $S^{\prime}$ is a proper subset of $S$, which contradicts the minimality of $S$. Hence $S$ has only one element which is a fixed point of $T$. The unicity of the fixed point follows from the fact that if $x=T x, y=T y$ then

$$
\|x-y\|=\|T x-T y\| \leqq \frac{\|x-T x\|}{2}+\frac{\|y-T y\|}{2}=0 \quad \text { i.e., } \quad x=y .
$$

THEOREM 2. Let T be a continuous mapping of a closed convex bounded set $K$ of a reflexive Banach space $X$ into itself and let $T$ have properties A and $\mathrm{B}$ over $K$. Then $T$ has a unique fixed point in $K$.

Proof. As in the previous theorem let $S$ be the minimal element in $X$ with respect to being closed, convex, bounded and invariant under $T$.

If $S$ contains only one element, the theorem is obvious. If not, by property $\mathrm{B}$, there exists $x \in S$ such that

$$
\|x-T x\|=r<\sup _{y \in S}\|y-T y\| .
$$

Let $P=\{x \in S:\|x-T x\| \leqq r\}$. If $x \in P$, then since

$$
\left\|T x-T^{2} x\right\| \leqq \frac{\|x-T x\|}{2}+\frac{\left\|T x-T^{2} x\right\|}{2}
$$

we have $\left\|T x-T^{2} x\right\| \leqq r$ which implies $T(P) \subset P$. Let $P^{\prime}=\mathrm{cl} \operatorname{co}(T P)$. If $z \in P^{\prime}$, then any one of the following three cases may arise:

(1) $z \in T P$ and since $T P \subset P$, hence $T z \in P^{\prime}$.

(2) $z=\sum_{1}^{n} \alpha_{i} T z_{i}, \alpha_{i} \geqq 0, \sum \alpha_{i}=1$ and $z_{i} \in P$.

$$
\begin{aligned}
\|z-T z\| & =\left\|\left(\sum \alpha_{i} T z_{i}\right)-T z\right\| \leqq \sum \alpha_{i}\left\|T z_{i}-T z\right\| \\
& \leqq \sum \alpha_{i}\left\{\frac{\|z-T z\|}{2}+\frac{\left\|z_{i}-T z_{i}\right\|}{2}\right\} \\
& \leqq \sum \alpha_{i}\left\{\frac{\|z-T z\|}{2}+\frac{r}{2}\right\}, \quad \text { since } z_{i} \in P, \\
& =\frac{\|z-T z\|}{2}+\frac{r}{2} .
\end{aligned}
$$

$\therefore\|z-T z\| \leqq r$ which implies $z \in P$ and hence $T z \in T P \subset P^{\prime}$. 
(3) $z$ is a limit point of $P^{\prime}$, in which case by the continuity of $T$ it follows that $z \in P$ and hence $T z \in P^{\prime}$.

Thus $P^{\prime}$ is a closed, convex subset of $S$ which is invariant under $T$ and, for every element $z$ of $P^{\prime},\|z-T z\| \leqq r$, which implies by (2) that $P^{\prime}$ is a proper subset of $S$. This contradicts the minimality of $S$. Hence $S$ contains only one element. This element is the unique fixed point of $T$, unicity being true as seen as in Theorem 1 .

If $X$ is a uniformly convex Banach space, then $K$ must have normal structure and as already noted [9, Theorem 5] normal structure in $K$ implies property $\mathrm{B}$ in $K$ for a mapping $T$ having property $\mathrm{A}$ on $K$ and mapping $K$ into itself. Hence we have

THEOREM 3. If $X$ is a uniformly convex Banach space and $K$ is a nonempty bounded closed convex subset of $X$ mapped into itself by a continuous mapping $T$ having property A on $K$, then $T$ has a unique fixed point in $K$.

REMARK. It should be noted that the reflexivity of $X$ can be replaced by weak compactness of $K$ in Theorem 2 .

COROllary. Let $K$ be a nonempty, closed, convex bounded subset of a Banach space $X$ and let $T$ be a continuous mapping of $K$ into itself having properties A and B over $K$. If $M$ is a weakly compact subset of $K$ such that $\mathrm{wc}\left\{T^{n} x\right\} \cap M \neq \varnothing$ for each $x \in K$, where wc $A$ denotes the weak closure of $A$, then $T$ has a unique fixed point in $K$.

Proof. If $H$ be any nonempty closed convex subset of $K$ and if $H$ is mapped into itself by $T$, then for $x \in H, \operatorname{wc}\left\{T^{n} x\right\} \cap M \neq \varnothing$. Hence $H \cap$ $M \neq \varnothing$. Using the weak compactness of $M$ we can now obtain a subset $K_{1}$ of $K$ minimal with respect to being nonempty, closed convex and invariant under $T$ and $K_{1} \cap M \neq \varnothing$. The rest of the proof follows similarly as in Theorem 2.

THEOREM 4. Let $T$ be a continuous mapping of a bounded compact convex subset $K$ of a Banach space $X$ into itself and let $T$ have property A over $K$. Then $T$ has a unique fixed point in $K$.

Proof. Let $S$ be the minimal element with respect to being closed, convex and invariant under $T$. Now compact convex subsets of a Banach space have normal structure [3]. Hence $T$ has property B on $S[9$, Theorem 5]. We now proceed as in Theorem 2 to complete the proof.

TheOREM 5. Let $X$ be a reflexive Banach space, $H$ a closed convex subset of $X$ and $K$ a nonempty bounded closed convex subset of $H$. Let $T$ be a 
continuous map of $K$ into $H$ such that

(1) $\|T x-T y\| \leqq \frac{1}{2}\{\|x-T x\|+\|y-T y\|\}, x, y \in K$;

(2) $T$ maps $\partial_{H} K$, the boundary of $K$ relative to $H$, into $K$;

(3) if $F$ be any closed convex subset of $K$ containing more than one element and if $G$ be a subset of $F$ such that $T G \subset F$ then there exists $x \in G$ such that $\|x-T x\|<\sup _{y \in F}\|y-T y\|$.

Then $T$ has a unique fixed point in $K$.

Proof. Let $\Gamma$ be the family of all closed convex subsets $F$ of $H$ such that $F \cap K \neq \varnothing$ and $T: F \cap K \rightarrow F$. Obviously $H \in \Gamma$. If $\left\{F_{\alpha}\right\}$ be any descending chain of subsets of $\Gamma$ then the weak compactness of each $F_{\alpha} \cap K$ implies that $F \cap K$, where $F=\bigcap F_{\alpha}$, is nonempty. Also $T: F \cap K \rightarrow F$ because $T: F_{\alpha} \cap K \rightarrow F_{\alpha}$ for each $\alpha$. Hence by Zorn's lemma there exists a minimal element $S$ in $\Gamma, S$ being minimal with respect to being closed, convex and such that $S \cap K \neq \varnothing$ and $T: S \cap K \rightarrow S$.

We may assume $\partial_{S} K \neq \varnothing$ for otherwise $S \subset K$ and $T: S \cap K \rightarrow S$ implies $T: S \rightarrow S$ and then the theorem would follow from Theorem 2 if one uses hypothesis (3). Now $\partial_{S} K \subset \partial_{H} K$. Hence $T: \partial_{S} K \rightarrow K$. Also $T: S \cap K \rightarrow S$. Hence $T$ maps $\partial_{S} K$ into $S \cap K$. If $S \cap K$ contains only one element $z$, then the nonemptiness of $\partial_{S} K \subset S \cap K$ implies that $z \in \partial_{S} K$ and $T: \partial_{S} K \rightarrow S \cap K$ implies that $T z=z$ which proves the theorem.

If $S \cap K$ contains more than one element, we will show that we arrive at a contradiction. By (3) there exists $x \in \partial_{S} K$ such that

$$
\|x-T x\|=r<\sup _{y \in S^{\prime} K}\|y-T y\| \text {. }
$$

Let $P=\{z \in S \cap K:\|z-T z\| \leqq r\}$ and let $P^{\prime}=\mathrm{cl} \operatorname{co}(T P)$, the closed convex hull of $T P$. Since $x \in \partial_{S} K, T x \in S \cap K$ i.e., $T x \in K$. Hence $T x \in P^{\prime} \cap K$.

Also let $z \in P^{\prime} \cap K$. We show that $T z \in P^{\prime}$. We do this as in Theorem 2. Here we give outlines only for the case when $z=T z_{1}, z_{1} \in P$. Now $z_{1} \in P$ implies that $z_{1} \in S \cap K$ and hence $z=T z_{1} \in S$. Also $z \in P^{\prime} \cap K$ gives $z \in K$. Hence $z \in S \cap K$ and we get

$$
\begin{aligned}
\|z-T z\| & =\left\|T z_{1}-T\left(T z_{1}\right)\right\| \leqq\left\|z_{1}-T z_{1}\right\|, \quad \text { by }(1), \\
& \leqq r .
\end{aligned}
$$

Thus $z \in P$ and hence $T z \in T P \subset P^{\prime}$.

Hence we find that $P^{\prime}$ is a closed convex subset of $S$ such that $P^{\prime} \cap K=$ $\varnothing$ and $T: P^{\prime} \cap K \rightarrow P^{\prime}$. Also as we have shown above $z \in P^{\prime} \cap K$ implies $\|z-T z\|<\sup _{y \in S \cap K}\|y-T y\|$. Hence $P^{\prime}$ is a proper subset of $S$ which is a contradiction.

COROLlaRY. Let $X$ be a reflexive Banach space and $K$ be a nonempty bounded closed convex subset of $X$. Also let $T$ be a continuous mapping of $K$ 
into $X$ such that

(1) $\|T x-T y\| \leqq \frac{1}{2}\{\|x-T x\|+\|y-T y\|\}, x, y \in K$;

(2) $T$ maps the boundary of $K$ into $K$;

(3) if $F$ be a closed convex subset of $K$ which contains more than one element and if $G$ be a subset of $F$ such that $T G \subset F$ then there exists $x \in G$ such that $\|x-T x\|<\sup _{y \in G}\|y-T y\|$.

Then $T$ has a unique fixed point in $K$.

A theorem similar to Theorem 6 for nonexpansive mappings may be seen in [12].

We now obtain a result analogous to an equivalent form of Theorem 2 for nonexpansive mappings. To this effect we first have the following proposition. We recall that $X$ is a reflexive Banach space and $K$ is a nonempty bounded closed convex subset of $X$.

Proposition 1. Let $T$ be a mapping of $K$ into itself having property A over $K$. Then the following statements are equivalent.

(a) $T$ has property B over $K$.

(b) For every nonempty bounded closed convex $T$-invariant subset $F$ of $K$ which contains more than one element there exists $x \in F$ such that $\sup _{r}\left\|x-T^{r} x\right\|<\sup _{z, y \in F}\|z-T y\|$. (This property would be referred to as property C.)

Proof. To show that (a) implies (b) it is sufficient to see that if $x$ be the element such that $\|x-T x\|<\sup _{y \in F}\|y-T y\|$ then the element $T x \in F$ would satisfy the hypothesis of (b) because $\left\|T x-T^{r}(T x)\right\| \leqq\|x-T x\|$ by the nature of $T$.

We now show that (b) implies (a). If possible let (a) be not true. Then there exists a nonempty bounded closed convex subset $F$ of $K$ which is $T$-invariant and contains more than one element such that, for every $x \in F$,

$$
\|x-T x\|=\sup _{y \in F}\|y-T y\|=0, \text { say. }
$$

Now consider $F^{\prime}=\mathrm{cl} \operatorname{co}(T F)$. For any two elements $z, w$ of $F^{\prime}$, it can be easily seen as in Theorem 2 that $\|z-T w\| \leqq p$. Also since $F^{\prime}$ is $T$-invariant and is contained in $F$, it follows that, for every $z \in F^{\prime}$,

$$
\sup _{r}\left\|z-T^{r} z\right\|=\sup _{z, u \in F^{\prime \prime}}\|z-T w\|
$$

and this is in contradiction with (b). Hence the proposition.

We now obtain the following theorem for nonexpansive mappings, the proof of which is essentially the same as that of Theorem 2 [1]. 
THEOREM 6. With the usual assumptions on $X$ and $K$ let $T$ be a nonexpansive mapping of $K$ into itself such that $T$ has property $\mathrm{C}$ over $K$. Then $T$ has a fixed point in $K$.

Combining Theorems 2 and 6 we can now write

THEOREM. Let $X$ be a reflexive Banach space and let $K$ be a nonempty bounded closed convex subset of $X$. Let $T$ be a continuous mapping of $K$ into itself such that $T$ has property $C$ over $K$. If $T$ satisfies either

$$
\|T x-T y\| \leqq \frac{1}{2}\{\|x-T x\|+\|y-T y\|\}, \quad x, y \in K,
$$

or

$$
\|T x-T y\| \leqq\|x-y\|, \quad x, y \in K .
$$

Then $T$ has a fixed point in $K$.

This gives rise to the following question.

Question. What hypothesis on $T$ is necessary besides property $\mathrm{C}$ over $K$, the other hypothesis on $X$ and $K$ being the same as above, in order to ensure the existence of a fixed point for $T$ ?

In the rest of the paper we make some observations on property $\mathrm{C}$.

Proposition 2. If $K$ has normal structure then $T$ has property $\mathrm{C}$ on $K$ where $T$ is a nonexpansive mapping of $K$ into itself.

Note. As mentioned earlier, that normal structure implies property B over $K$ for a mapping $T$ having property $\mathrm{A}$ over $K$, or equivalently by Proposition 1, property $\mathrm{C}$ over $K$ has been proved in [9].

Proof. We proceed exactly as in Proposition 1. That the converse of the above proposition is not true can be easily seen from the following example [13].

EXAMPLE. Let $B$ be the space isomorphic to the Hilbert space with norm defined (for $x \in H$ ) by $\|x\|=\sup \left\{\frac{1}{2}\|x\|_{H},\left|x_{n}\right|\right\}$. Then $K=\left\{x:\|x\|_{H} \leqq 1\right.$ and $x_{i} \geqq 0$ for all $\left.i\right\}$ does not have normal structure. It is easy to define nonexpansive mappings $T$ on $K$ for which property $\mathrm{C}$ is true.

A connection similar to that of Proposition 2 exists between the concept of diminishing orbital diameters and property $\mathrm{C}$. The proof of the following proposition is similar to that of Proposition 1.

Proposition 3. For a nonexpansive mapping $T$ of $K$ into itself, $T$ has diminishing orbital diameters over $K$ implies $T$ has property $\mathrm{C}$ over $K$.

Note. It must be noted here [9] that, for a continuous mapping $T$ having property $\mathrm{A}$ over $K$, the statements $T$ has diminishing orbital diameters over $K$ and $T$ has property $\mathrm{C}$ over $K$ are equivalent. That the converse of Proposition 3 is not true can be easily seen. 
REMARK. After this paper was communicated, Theorem 3 was proved recently by P. Soardi [Boll. Un. Mat. Ital. 4 (1971), 841-845]. The author would like to thank the referee for his suggestions.

\section{REFERENCES}

1. L. P. Belluce and W. A. Kirk, Fixed point theorems for certain classes of nonexpansive mappings, Proc. Amer. Math. Soc. 20 (1969), 141-146. MR 38 \#1663.

2. F. E. Browder, Nonexpansive nonlinear operators in a Banach space, Proc. Nat. Acad. Sci. U.S.A. 54 (1965), 1041-1044. MR 32 \#4574.

3. R. E. DeMarr, Common fixed points for commuting contraction mappings, Pacific J. Math. 13 (1963), 1139-1141. MR 28 \#2446.

4. D. Göhde, Zum Prinzip der kontraktiven Abbildung, Math. Nachr. 30 (1965), 251258. MR 32 \#8129.

5. K. Goebel, An elementary proof of the fixed-point theorem of Browder and Kirk, Michigan Math. J. 16 (1969), 381-383. MR 40 \#4831. 76.

6. R. Kannan, Some results on fixed points, Bull. Calcutta Math. Soc. 60 (1968), 71-

7. — Some results on fixed points. II, Amer. Math. Monthly 76 (1969), 405-408.

8. - Some results on fixed points. III, Fund. Math. 70 (1971), 169-177.

9. - Some results on fixed points. IV, Fund. Math. 74 (1972), 181-187.

10. W. A. Kirk, A fixed point theorem for mappings which do not increase distances, Amer. Math. Monthly 72 (1965), 1004-1006. MR 32 \#6436.

11. V. Smulian, On the principle of inclusion in the space of the type (B), Mat. Sb. 5 (47) (1939), 327-328. (Russian) MR 1, 335.

12. W. A. Kirk, Fixed point theorems for nonlinear nonexpansive and generalized contraction mappings, Pacific J. Math. 38 (1971), 89-95.

13. L. P. Belluce, W. A. Kirk and E. F. Steiner, Normal structure in Banach spaces, Pacific J. Math. 26 (1968), 433-440. MR 38 \#1501.

Department of Mathematics, Purdue University, Lafayette, Indiana 47907

Current address: Mathematics Department, University of Missouri, St. Louis, Missouri 63121 